\title{
EFEITO DA MELATONINA SOBRE A MORFOFISIOLOGIA CARDÍACA DE RATOS INDUZIDOS A HIPERLIPIDEMIA
}

\section{ARTIGO ORIGINAL}

FERREIRA, Fernanda Amorim ${ }^{1}$

PARAÍBA, Isabella Maria Rios ${ }^{2}$

ELIAS, Laíse de Souza ${ }^{3}$

FERREIRA, Fernanda Amorim. PARAÍBA, Isabella Maria Rios. ELIAS, Laíse de Souza. Efeito da melatonina sobre a morfofisiologia cardíaca de ratos induzidos a hiperlipidemia. Revista Científica Multidisciplinar Núcleo do Conhecimento. Ano 05, Ed. 10, Vol. 13, pp. 130-143. Outubro de 2020. ISSN: 2448-0959, Link de acesso: https://www.nucleodoconhecimento.com.br/nutricao/efeito-da-melatonina

\section{RESUMO}

As hiperlipidemias são classificadas como alterações metabólicas e estão relacionadas com doenças cardiovasculares, as quais se destacam a doença aterosclerótica coronariana. As doenças cardiovasculares têm notável participação nas taxas de mortalidade a nível mundial e em virtude disso, vem sendo alvo de pesquisas que buscam inovação terapêutica. A melatonina é um hormônio produzido em fase de escuridão ambiental pela glândula pineal que desempenha função antioxidante, hipolipemiante, anti-inflamatória, entre outras, demonstrando assim, ser uma potente molécula no tratamento de doenças cardiovasculares. Assim, o objetivo

\footnotetext{
${ }^{1}$ Graduada em Nutrição pelo Centro Universitário Uninassau.

${ }^{2}$ Mestranda em Nutrição Comunitária e Saúde Pública pela FCNAUP Graduada em Nutrição pelo Centro Universitário Uninassau.

3 Orientadora. Doutorado em Biociência Animal. Mestrado em Biociência Animal. Especialização em Morfologia. Graduação em Licenciatura em Ciências Biológicas.
} 
deste trabalho consiste em avaliar o efeito da melatonina sobre o estresse oxidativo e parâmetros bioquímicos em ratos induzidos à hiperlipidemia. Para isso, foram utilizados 15 ratos albinos (Rattus norvegicus albinus) machos, com 150 dias de idade, procedentes do biotério do departamento de Morfologia e Fisiologia Animal da Universidade Federal Rural de Pernambuco. Esses animais foram mantidos em gaiolas, com alimentação e água ad libitum, temperatura de $22^{\circ} \mathrm{C}$ e iluminação artificial que estabelecerá um fotoperíodo de 12 horas claro e 12 horas escuro (ciclo invertido). Em conclusão, estudos demonstraram que a melatonina tem efeitos na redução da peroxidação lipídica, níveis de creatina quinase (CK) bem como na diminuição da glutationa reduzida. Como uma droga barata e bem tolerada, a melatonina pode ser uma nova opção terapêutica para doenças cardiovasculares e indivíduos com hiperlipidemia.

Palavras-chave: Aterosclerose, melatonina, coração, hiperlipidemia, estresse oxidativo.

\section{INTRODUÇÃO}

As hiperlipidemias são alterações metabólicas que ocorrem quando os níveis de lipídios circulantes ultrapassam os limites da normalidade na corrente sanguínea (BEVILACQUA et al., 2007). Essa produção desregulada pode acarretar sérios problemas, entre eles, as doenças cardiovasculares (DCVs), como hipertensão arterial, aterosclerose, além de danos em diferentes órgãos e tecidos, caracterizando assim, um grave problema de saúde pública (EMET et al., 2016).

As DCVs têm notável participação nas taxas de mortalidade a nível mundial e, em virtude disso, vem sendo alvo de pesquisas que buscam inovações terapêuticas. (SIMKO et al., 2016). Dessa forma, nas últimas décadas, a busca de novos medicamentos para o tratamento da hiperlipidemia tem valorizado o papel de substâncias que atuem no controle do aumento do perfil lipídico e da inflamação, além de proteger o endotélio vascular contra o estresse oxidativo (JOCKERS et al., 2016). Dentre essas substâncias, a melatonina tem recebido bastante repercussão para o tratamento de lesões em diversos órgãos, inclusive o coração (YANG et al., 2014). 
A melatonina ( $\mathrm{N}$-acetil-5-metoxitriptamina) (MEL) é um hormônio neuroendócrino produzido pela glândula pineal (HARDELAND et al., 2006). A sua secreção é regulada pelo ciclo luz/escuridão ambiental através do núcleo supraquiasmático (SKENE et al., 2006). Sendo sua biossíntese iniciada no período noturno, a partir de fotorreceptores da retina que enviam sinal para a glândula pineal, estimulando a produção e liberação da melatonina (GOOLEY et al., 2003). O mecanismo de ação da MEL depende de receptores de membrana denominados MT1, MT2 e MT3, sendo estes expressos tanto individualmente como em conjunto em vários tecidos e órgãos, sendo os dois primeiros expressos tanto no coração quanto em artéria de ratos (CAHILL; GRACE; BESHARSE, 1991) (LI; ZHANG; TANG, 2013).

Por apresentar receptores amplamente distribuídos pelo corpo, a MEL pode ser responsável por uma série de eventos fisiológicos (FARÍAS; ZEPEDA; CALAF, 2012). Inicialmente sua função estava restrita a regulação do ciclo sono/vigília, (SUN; HUANG; QU, 2015) entretanto, com o passar dos anos outras funções também foram relatadas, como, o seu papel antioxidante e anti-inflamatório, na regulação do metabolismo de lipídios e glicose, (AGIL et al., 2011) (ESPINO; PARIENTE; RODRIGUEZ, 2011) bem como seu efeito cardioprotetor, tendo um papel importante sobre doenças cardíacas (TAN et al., 1998), como lesão de isquemia-reperfusão miocárdica, aterosclerose, hipertensão e insuficiência cardíaca. (FAVERO et al., 2012) (BENOVA et al., 2013) (DOMINGUEZ; ABREU-GONZALEZ; REITER, 2014).

Estudos em ratos verificaram que a MEL produziu efeito cardioprotetor frente a cardiotoxicidade induzida por agente quimioterapêutico e, recentemente foi reiterado a amplitude do efeito protetor cardíaco exercido pela MEL (SUN; GUSDON; QU, 2016). Além disto, foi verificado que a melatonina foi eficiente em reduzir os efeitos da isquemia induzida no coração de ratos (YU et al., 2014), redução da frequência cardíaca, redução dos níveis da proteína creatina quinase, (FAVERO et al., 2016), preservação da microestrutura do cardiomiócito, (LIN et al., 2018), redução de arritmias cardíacas e da oxidação lipídica consequente de processos isquêmicos cardíacos, (TARE et al., 2014), além de melhorar a função cardíaca e o fluxo sanguíneo (ZHANG et al., 2017). 
Tendo em vista o crescente aumento de portadores da hiperlipidemia (KANG; KOH; LEE, 2011) e consequentemente, doenças cardiovasculares (HARDELAND, 2015) e que a melatonina desempenha função antioxidante, (PANDI-PERUMAL et al., 2013) (COMMENTZ; HELMKE, 1995), (REITER et al., 2013) anti-inflamatória e hipolipemiante (ALLEGRA et al., 2003), faz-se necessário estudar a eficácia do efeito da melatonina na morfofisiologia cardíaca em ratos induzidos à hiperlipidemia. Dessa forma, esta pesquisa pode contribuir para o desenvolvimento de métodos terapêuticos complementares no tratamento da hiperlipidemia, bem como, das doenças cardiovasculares. O presente estudo teve como objetivo, avaliar o efeito da melatonina sobre a morfofisiologia cardíaca de ratos induzidos à hiperlipidemia.

\section{MATERIAIS E MÉTODOS}

\subsection{GRUPOS EXPERIMENTAIS}

Foram utilizados 15 ratos albinos (Rattus norvegicus albinus) machos, com 150 dias de idade, procedentes do biotério do departamento de Morfologia e Fisiologia Animal da Universidade Federal Rural de Pernambuco. Esses animais foram mantidos em gaiolas, com alimentação e água ad libitum, temperatura de $22^{\circ} \mathrm{C}$ e iluminação artificial que estabeleceu um fotoperíodo de 12 horas claro e 12 horas escuro (ciclo invertido). Os animais foram pesados e divididos aleatoriamente em três grupos experimentais: GC: ratos sem indução a hiperlipidemia; GI: ratos induzidos a hiperlipidemia e tratados com placebo; GM: ratos induzidos à hiperlipidemia e tratados com melatonina.

\subsection{INDUÇÃO DA HIPERLIPIDEMIA}

Para induzir a hiperlipidemia foi administrado durante trinta dias, por via intraperitoneal, Triton WR 1339, também conhecido como tyloxapol, um detergente não aniônico de estrutura polimérica, na dose de $400 \mathrm{mg} / \mathrm{Kg}$ de peso corporal, dissolvido em $\mathrm{NaCl}$ à $0,9 \%$. Esta substância tem sido utilizada em trabalhos experimentais por ser capaz de causar hiperlipidemia em animais. Para acompanhamento da hiperlipidemia, após o terceiro dia de indução foram realizadas

RC: 62419

Disponível em: https://www.nucleodoconhecimento.com.br/nutricao/efeito-da-melatonina 
análises bioquímicas, para tal, foram utilizados kits da Labtest $\Theta$, seguindo os catálogos especificados: LDL, HDL, triglicerídeos e colesterol total. Os animais não induzidos à hiperlipidemia (GC) receberam da mesma forma, doses equivalentes de solução salina.

\subsection{ADMINISTRAÇÃO DA MELATONINA}

A melatonina foi administrada via oral (VO), para isso, $10 \mathrm{mg} / \mathrm{Kg}$ de melatonina foi dissolvida em 200 microlitros de álcool. Em seguida, no horário das sete horas da manhã esta solução foi diluída na água de beber dos animais, vale salientar que as garrafas foram revestidas por papel alumínio para proteger o conteúdo da luminosidade.

\subsection{ANÁLISE BIOQUÍMICA}

As amostras de sangue foram coletadas nos períodos antes da indução e após o tratamento. Para análise antes do período da indução, os ratos foram mobilizados por contensor mecânico e o sangue coletado por punção caudal lateral com o uso de cateter (24G). Já para análise após indução e seu respectivo tratamento, o sangue foi coletado por punção cardíaca. O material foi centrifugado, o sobrenadante acondicionado em Eppendorf e mantido em freezer a $-20^{\circ} \mathrm{C}$ até o momento das dosagens. Para tal, foi utilizado o kit da Labtest $\AA$, seguindo o catálogo específico: proteína CK (133-1/500).

\subsection{ANÁLISE DO ESTRESSE OXIDATIVO: PEROXIDAÇÃO LIPÍDICA E GLUTATIONA REDUZIDA}

A peroxidação lipídica foi estimada através da medida dos níveis de substâncias reativas de ácido tiobarbitúrico (TBARS), enquanto a glutationa reduzida (GSH) foi determinada através da mensuração de grupamentos sulfidrilas não protéicos. Para isso, fragmentos do coração foram macerados em $\mathrm{KCl}, 1,15 \%$ numa proporção de 10 $\mathrm{ml} / 1 \mathrm{~g}$ até completa homogeneização do material coletado. $\mathrm{O}$ homogenato foi transferido para um tubo de ensaio, ao qual foi adicionado $2 \mathrm{~mL}$ de regente $(0,375 \%$ 
ácido tiobarbitúrico e $75 \%$ ácido tricloroacético) para cada $\mathrm{mL}$ da mistura. Os tubos em duplicata foram lacrados e aquecidos em banho maria $\left(100{ }^{\circ} \mathrm{C}\right)$ durante 15 minutos. O sobrenadante foi separado e a absorbância medida a $535 \mathrm{~nm}$.

\subsection{PROCEDIMENTOS ÉTICOS}

O experimento foi desenvolvido de acordo com os Princípios Éticos na Experimentação com Animais, segundo a Lei n o 11.794 de 8 de outubro de 2008, tendo sido o projeto aprovado pela Comissão de Ética no Uso de Animais (CEUA) da Universidade Federal Rural de Pernambuco (UFRPE - processo de número 23082.015842/2014) (ANEXO A).

\subsection{ANÁLISE ESTATÍSTICA}

As análises estatísticas foram realizadas por meio do método não paramétrico de Kruskal-Wallis, onde as médias foram comparadas pelo teste de Wilcoxon-Mann Whitney, 95\% de significância.

\section{RESULTADOS E DISCUSSÃO}

Os danos oxidativos induzidos nas células e tecidos têm sido relacionados a processos fisiológicos e com a etiologia de várias doenças, como envelhecimento, e doenças crônicas, tais como diabetes, câncer e aterosclerose, onde frequentemente se observa o aumento do conteúdo das espécies reativas de oxigênio (EROs) mais importantes no contexto biológico (ROSEN et al., 2001) (HIGASSHI et al., 2014).

As análises dos níveis de TBARS no tecido cardíaco após indução à hiperlipidemia revelaram valores elevados quando comparados ao grupo controle. Porém, o tratamento com a melatonina atenuou os níveis de TBARs, apresentando valores semelhantes ao GC (Figura 1). A análise tecidual dos níveis de GSH no grupo induzido e tratado com melatonina foram superiores aos animais do grupo Gl, demonstrando também valores similares aos do grupo controle (Figura 2). Com isso, percebemos que a melatonina foi capaz de reduzir os danos oxidativos. 
Este hormônio apresenta a capacidade de sequestrar as espécies reativas de oxigênio (EROs) e de nitrogênio (ERN), atuando na prevenção ao dano oxidativo (ALVES et al., 2014) além de estimular a expressão de enzimas antioxidantes (PANDI-PERUMAL et al., 2013) através do crescimento da expressão da via Keap1- Nrf2 (MANCHESTER et al., 2015).

ONK (2016), constatou que o uso de $20 \mathrm{mg} / \mathrm{kg}$ de melatonina resultou no decréscimo dos níveis de TBARS. Da mesma forma, Debosree Ghosh (2015), evidenciou que a dose mínima de $10 \mathrm{mg} / \mathrm{kg}$ de melatonina foi necessária para proteção contra o aumento da peroxidação lipídica nos glóbulos vermelhos de ratos.

Inúmeros trabalhos são relatados demonstrando o efeito isolado da melatonina na promoção dos níveis de GSH, por exemplo, foi demonstrado uma elevação dos níveis de GSH em animais com danos hepáticos induzidos por tioacetamida (TAA) e tratadas com melatonina (10 mg/kg) Czechowska (2015) e Goc (2017), observaram níveis elevados de GSH no tecido hepático dos animais tratados com $10 \mathrm{mg} / \mathrm{kg}$ de MEL isolada ou em combinação com o nitroprussiato de sódio (SNP), um fármaco antihipertensivo com efeitos tóxicos, em comparação com a administração de SNP isolado.

Em nosso estudo, o grupo induzido a hiperlipidemia e tratado com melatonina, demonstrou redução dos níveis da proteína $\mathrm{Ck}$ em comparação com o grupo hiperlipidêmico (Gl), porém, em relação ao grupo controle não foi observado nenhuma alteração significativa (Tabela 1).

A creatinoquinase (CK) é uma enzima que desempenha importante papel na geração de energia para o metabolismo muscular. Está presente, predominantemente no tecido muscular, mas é também encontrada no tecido cerebral (BRANCACCIO et al., 2008). Dentre as moléculas utilizadas como marcador de dano cardíaco, a creatina quinase (CK) é frequentemente descrita como o melhor marcador indireto de dano ao tecido muscular (FOSCHINI et al., 2017). Essa redução dos níveis de CK pode ser atribuída aos efeitos de estabilização de membrana dada pela MEL. Estudos in vitro demonstram que a MEL é capaz de inibir a internalização da fosfatidilserina, 
expressão de Bax e xantina oxidase, causando assim, estabilização da bicamada lipídica (KOCIC et al., 2017).

Ghaeli (2015), refere que em pacientes com infarto do miocárdio com supradesnivelamento do segmento ST submetidos à intervenção coronariana percutânea primária, a administração de melatonina mais o tratamento padrão diminuiu consideravelmente o nível de creatina quinase-MB. Além disso, a MEL ainda apresenta efeitos anti-inflamatórios e anti apoptóticos, podendo assim, atuar no controle de doenças cardiovasculares (CHEN et al., 2016).

\section{CONCLUSÃO}

Em conclusão, estudos demonstraram que a melatonina tem efeitos na redução da peroxidação lipídica, níveis de creatina quinase (CK) bem como no aumento da glutationa reduzida em ratos induzidos à hiperlipidemia.

\section{REFERÊNCIAS}

AGIL, A, Navarro-Alarcon M, Ruiz R, et al. Beneficial effects of melatonin on obesity and lipid profile in young Zucker diabetic fatty rats. J Pineal Res 2011; 50:207-212.

ALLEGRA, M.; REITER, R. J.; TAN, D. X.; et al. The chemistry of melatonin's interaction with species. J. Pineal Res., v.34, n.1, p. 1-10, 2003.

ALVES, R.S.C. et al. A melatonina e o sono em crianças. Pediatria. v. 1, p. 587594,2004 .

BRANCACCIO, P.; Maffulli, N.; Buonauro, R. Serum enzyme monitoring in sports medicine. Clin Sports Med 2008. 27:1-1.

BENOVA, T, Viczenczova C, Radosinska J, et al. Melatonin attenuates hypertensionrelated proarrhythmic myocardial maladaptation of connexin-43 and propensity of the heart to lethal arrhythmias. Can J Physiol Pharmacol 2013; 91:633-639. 
BEVILACQUA, MR, GIMENO SGA, BEVILACQUA MR, Matsumura LK, et al. Hiperlipidemias e Fatores Dietéticos: Estudo Transversal Entre Nipo-Brasileiros. Arq Bras Endocrinol Metab. 2007 mar/out; 51/4: 547-558.

CAHILL, GM, Grace MS, Besharse JC. Rhythmic regulation of retinal melatonin: metabolic pathways, neurochemical mechanisms, and the ocular circadian clock. Cell Mol Neurobiol 1991; 11:529-560.

CHEN, S,J. et al. Melatonin enhances interleukin-10 expression and suppresses chemotaxis to inhibit inflammation in situ and reduce the severity of experimental autoimmune encephalomyelitis. Int Immunopharmacol. v.31, p.169-77, 2016.

COMMENTZ, J. C.; HELMKE, K. Precocious puberty and decreased melatonin1583 secretion due to a hypothalamic hamartoma. Horm.Res. v.44, n. 6, p. 271-275,1584 1995.

CZECHOWSKA, G. et al. Protective effects of melatonin against thioacetamide induced liver fibrosis in rats. J Physiol Pharmacol. v.66, p.567-79, 2015 .

DEBOSREE Ghosh; Sudeshna Paul, Aindrila Chattopadhyay, Debasish Bandyopadhy. Melatonin and aqueous curry leaf extract in combination protects against lead induced oxidative stress mediated injury to rat heart: a new approach. Journal of Pharmacy Research 2015,9(12),618-634.

DOMINGUEZ -Rodriguez A, Abreu-Gonzalez P, Reiter RJ. The potential usefulness of serum melatonin level to predict heart failure in patients with hypertensive cardiomyopathy. Int J Cardiol 2014; 174:415-417.

EMET M, Ozcan H, Ozel L, Yayla M, Halici Z, Hacimuftuoglu A. A review of melatonin, its receptors and drugs. Eur J Med. 2016; 48(2):135-41. 
ESPINO J, Pariente JA, Rodriguez AB. Role of melatonin on diabetes-related metabolic disorders. World J Diabetes 2011; 2:82-91.

FARIAS JG, Zepeda AB, Calaf GM. Melatonin protects the heart, lungs and kidneys from oxidative stress under intermittent hypobaric hypoxia in rats. Biol Res. 2012; 45(1):81-5.

FAVERO G, Rodella LF, Reiter RJ, et al. Melatonin and its atheroprotective effects: a review. Mol Cell Endocrinol 2014; 382:926-937.

FAVERO G, Franceschetti L, Buffoli B, Moghadasian MH, Reiter RJ, Rodella LF, et al. Melatonin: Protection against age-related cardiac pathology. Ag Res Rev. 2016; 22: S1568-1637. 16.

FOSCHINI D, Prestes J, Charro MA. Relação entre exercício físico, dano muscular e dor muscular de início tardio. Rev Bras Cineantropom Desempenho Hum 2007;9(1):101-6.

GHAELI P, Vejdani S, Ariamanesh A, et al. Effect of melatonin on cardiac injury after primary percutaneous coronary intervention: a randomized controlled trial. Iran $\mathbf{J}$ Pharm Res 2015; 14:851-855.

GOC, Z. et al. Protective effects of melatonin on the activity of SOD, CAT, GSHPx and GSH content in organs of mice after administration of SNP. Chin J Physiol. v.1, p. 28-34 2017.

GOOLEY, JJ, Lu J, Fischer D, Saper CB. A broad role for melanopsin in nonvisual photoreception. J Neurosci 2003; 23:7093-7106.

HARDELAND, R, Pandi-Perumal SR, Cardinali DP. Melatonin. Int J Biochem Cell Biol. 2006; 38:313-316.

HARDELAND, R. Antioxidative protection by melatonin: multiplicity of mechanisms from radical detoxification to radical avoidance. Endocrine. v. 27, p. 111-118, 2015. 
HIGASSHI; $\mathrm{T}$ Maruhashi, $\mathrm{K}$ Noma, $\mathrm{Y}$ Kihara Oxidative stress and endothelial dysfunction: Clinical evidence and therapeutic implications, Trends in Cardiovascular Medicine Volume 24, Issue 4, May 2014, Pages 165-169.

JOCKERS, R, Delagrange P, Dubocovich ML, Markus RP, Renault N, Tosini G, et al. Update on melatonin receptors: IUPHAR Review. Br J Pharmacology. 2016; 173:2702-25.

KANG, J. W.; KOH, E. J.; LEE, S. M. Melatonin protects liver against ischemia and reperfusion injury through inhibition of toll-like receptor signaling pathway. J Pineal Res. v.50, p. 403-411, 2011.

KOCIC, G., Tomovic, K., Kocic, H., et al., 2017. Antioxidative, membrane protective and antiapoptotic effects of melatonin, in silico study of physico-chemical profile and efficiency of nanoliposome delivery compared to betaine. RSC Adv. 7, 1271-1281.

LI, X.; ZHANG, M.; TANG, W. Effects of melatonin on streptozotocin-induced retina neuronal apoptosis in high blood glucose rat. Neurochem Res. v.38, p.669- 76, 2013.

LIN, X, Zhao T, Lin $\mathrm{CH}$ et al. Melatonin provides protection against heat strokeinduced myocardial injury in male rats. J Pharm Pharmacol. 2018 Jun;70(6):760-767.

MANCHESTER LC, et al. Melatonin: an ancient molecule that makes oxygen metabolically tolerable. J Pineal Res. v.59, p. 403-19, 2015

ONK D, et al. Melatonin Attenuates Contrast-Induced Nephropathy in Diabetic Rats: The Role of Interleukin-33 and Oxidative Stress. Mediators Inflamm. v.2016, p. 50828, 2016.

PANDI-PERUMAL, S. R. et al. Melatonin antioxidative defense: therapeutical implications for aging and neurodegenerative processes. Neurotox Res. v.23, p.267300, 2013. 
REITER, R. J. et al. Melatonin as an antioxidant: biochemical mechanisms and pathophysiological implications in humans - Acta Biochim. Pol.v. 50, p. 1129-1146, 2003.

RODRIGUEZ C, et al. Regulation of antioxidant enzymes: a significant role for melatonin. J Pineal Res. 2004;36(1):1-9.

ROSEN, _P P Nawroth, G King, W Möller, H J Tritschler, L Packer. The role of oxidative stress in the onset and progression of diabetes and its complications: a summary of a Congress Series sponsored by UNESCO-MCBN, the American Diabetes Association and the German Diabetes Society - May-Jun 2001;17(3):189-212.

SIMKO F, Baka T, Paulis L, Reiter RJ. Elevated heart rate and non dripping heart rate as potential targets for melatonin: a review. J Pineal Res. 2016; 61:127-37.

SKENE, DJ, Arendt J. Human circadian rhythms: physiological and therapeutic relevance of light and melatonin. Ann Clin Biochem. 2006; 43:344-353.

SUN H, Huang FF, Qu S. Melatonin: a potential intervention for hepatic steatosis. Lipids Health Dis. 2015; 14:75.

SUN H, Gusdon AM, Qu S. Effects of melatonin on cardiovascular diseases: progress in the past year. Curr Opin Lipidol. 2016; 27(4):408-13.

TAN DX, Manchester LC, Reiter RJ, et al. Ischemia/reperfusion-induced arrhythmias in the isolated rat heart: prevention by melatonin. J Pineal Res. 1998; 25:184-191.

TARE M, Parkington HC, Wallace EM et al. Maternal melatonin administration mitigates coronary stiffness and endothelial dysfunction, and improves heart resiliência to insult in growth restricted lambs. 2014

YANG Y, Sun Y, Yi W, Li Y, Fan C, Xin Z, et al. A review of melatonin as a suitable antioxidant against myocardial ischemia-reperfusion injury and clinical heart diseases. J Pineal Res. 2014;57(4):357-66. doi: 10.1111/jpi.12175. 
YU L, Sun Y, Cheng L, Jin Z, Yang Y, Zhai M, et al. Melatonin receptor mediated protection against myocardial ischemia/reperfusion injury: role of SIRT1. J Pineal Res. $2014 ; 57(2): 228-38$.

ZHANG, Y. et al. Melatonin protects against arsenic trioxide-induced liver injury by the upregulation of Nrf2 expression through the activation of PI3K/AKT pathway. Oncotarget. 2017.

\section{ANEXO}

Figura 1: Avaliação dos níveis de TBARS (nmol/mg de proteína) no tecido cardíaco dos animais nos diferentes grupos experimentais) *Médias seguidas pela mesma letra não diferem significativamente entre si pelo teste de Kruskal-Wallis com post-hoc Dunn $(p<0,05)$. GC: controle; $G$ : induzido à hiperlipidemia; $G I M$ : induzido à hiperlipidemia

e tratado

com melatonina.
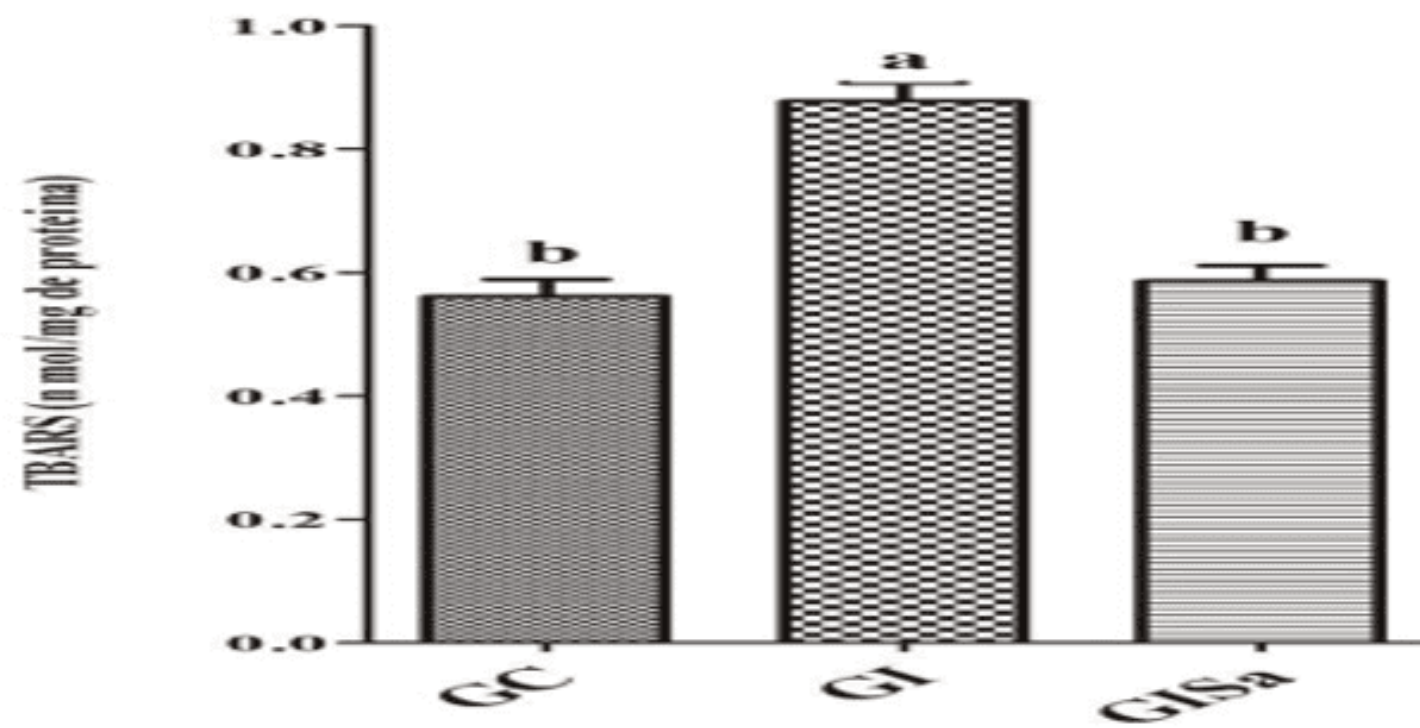

Figura 2. Avaliação dos níveis de GSH (nmol/mg de proteína) no tecido cardíaco dos animais nos diferentes grupos experimentais) *Médias seguidas pela mesma letra não diferem significativamente entre si pelo teste de Kruskal-Wallis com post-hoc Dunn 
$(p<0,05)$. GC: controle; $G$ : induzido à hiperlipidemia; GIM: induzido à hiperlipidemia e tratado com melatonina.

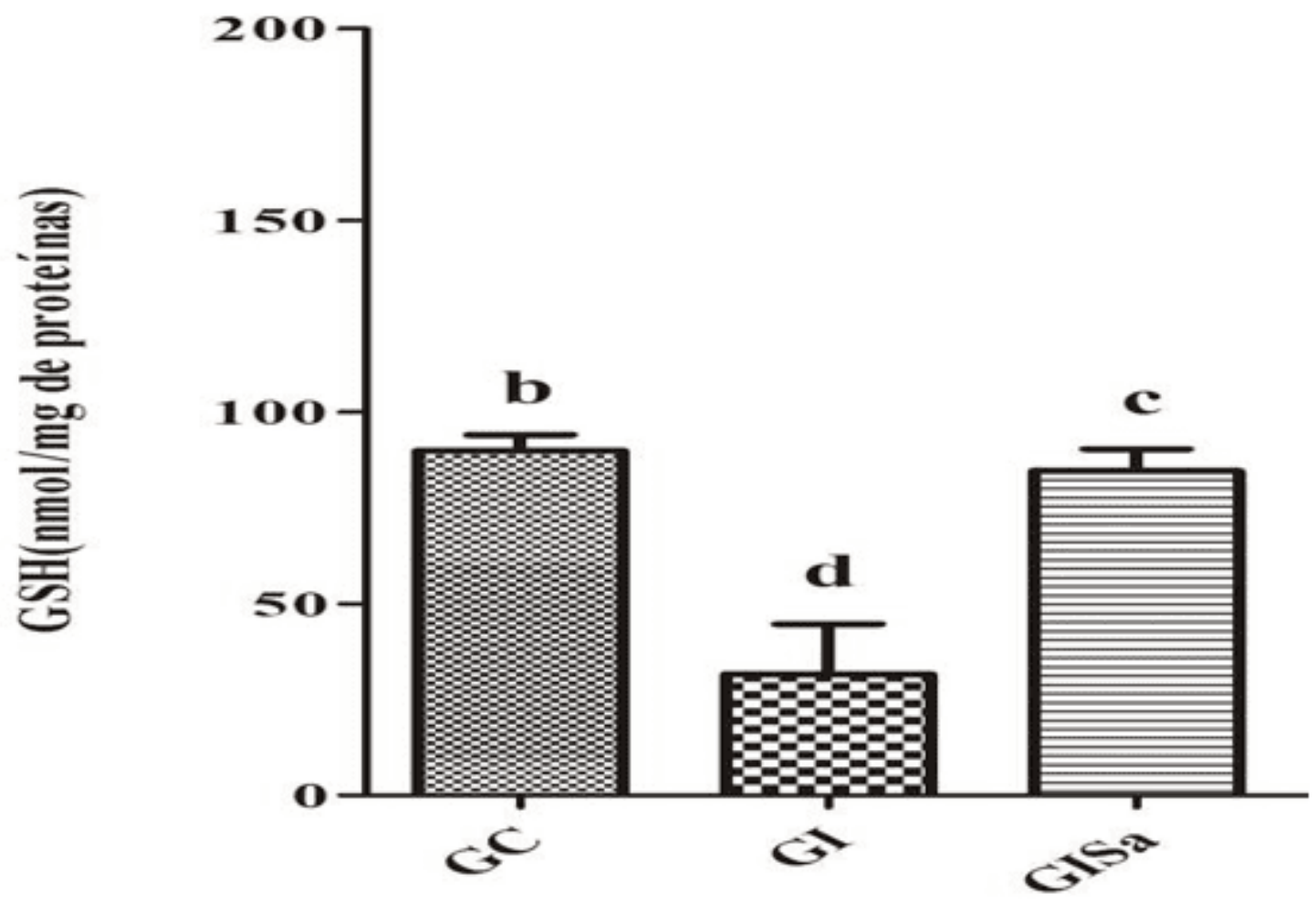

Tabela 1: Análise bioquímica dos grupos experimentais

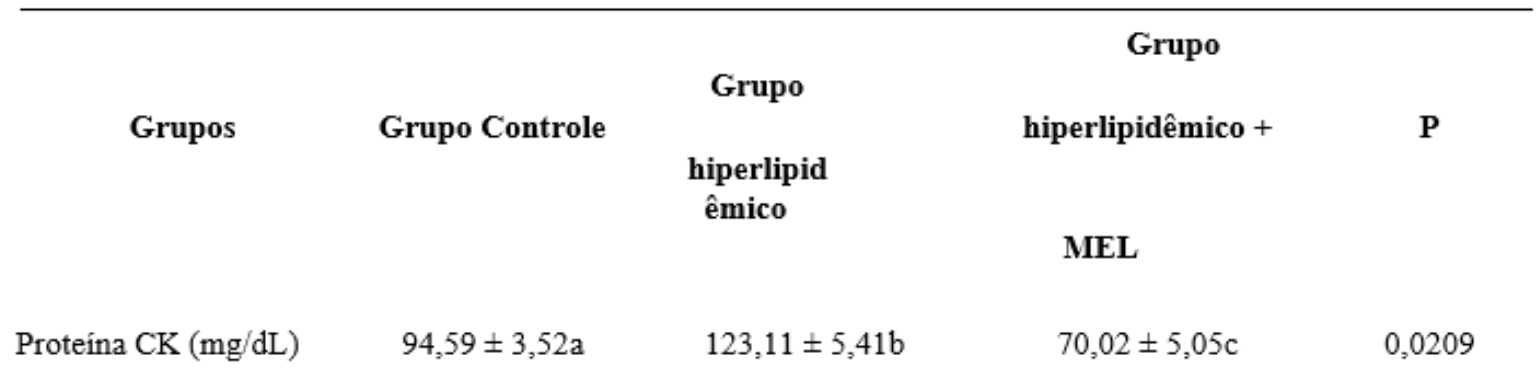

Tabela 1: Análise da proteína CK 
Enviado: Agosto, 2020.

Aprovado: Outubro, 2020. 